\title{
Montaigne: ein Medizinprogramm im sechzehnten Jahrhundert
}

Von René Bernoulli

Es ist eine leidige Sache um die Kritik an der zeitgenössischen Medizin. Trotz der Reichhaltigkeit der diagnostischen und therapeutischen Möglichkeiten tönt es laut, die Gegenwartsmedizin stecke in einer Krise, oder sie befinde sich in einem Übergangsstadium. Nach der deontologischen Standesliteratur zu urteilen, betrifft die anscheinend wenig erfreuliche Situation der gegenwärtigen Heilkunde in gleichem Maße die Forschung, die Klinik und die tägliche Sprechstunde, den Studenten wie den Dozenten.

Gewiß fehlt es nicht an Remedurvorschlägen. So wohlmeinend die Reformpläne auch sein mögen, so können sie bei der Widersprüchlichkeit, mit der sie sich selbst oft gegenüberstehen, schwerlich weiterhelfen. Unwidersprochen ist einzig die Ansicht, daß, soweit Mißstände effektiv vorliegen, in erster Linie die Patienten die Leidtragenden sind. Aber auch der Arzt muß unter Umständen der gegenwärtigen Lage seinen Tribut zollen. Er wird jedenfalls unserem Jubilar, dem die vorliegenden Publikationen gewidmet sind, zweifelsohne zustimmen, wenn dieser den heutigen Praktiker in einer «an richtungsweisenden Zielen so armen Zeit» tätig sieht ${ }^{1}$.

Nun lehrt uns die Medizingeschichte, daß es auch früher schon Epochen gab, die als «Krisenzeiten» der Medizin zu bezeichnen sind. Auch damals hatte es der einzelne Arzt oft schwer, echten Fortschritt von ephemeren Modeströmungen, Bewährtes von übernommenen Übelständen zu unterscheiden. Eine solche Ära, die als Paradigma für eine Übergangszeit gelten kann, ist die Renaissance. Dieses kulturgeschichtlich höchst bemerkenswerte Zeitalter war allerdings für den damaligen Arzt, analog zu heute, auch eine eher spannungsgeladene Epoche. Ein besonders sprechendes Beispiel liefert der «Fall» Vesal:

Da hatten doch die Humanistenärzte - unter denen der an der Pariser Medizinischen Fakultät wirkende Basler Wilhelm Cop (um 1460-1532) besonders zu erwähnen ist ${ }^{2}$ - die vorwiegend auf Galen beruhende Medizin dank ihren Übersetzungen der nunmehr zugänglich gewordenen Originalien der Fachwelt in einer bis dahin schier unbekannten Authentizität nähergebracht. Und nun kommt ein noch nicht einmal Dreißigjähriger - es war im Jahre 1543 - namens Andreas Vesalius und stellt mit seinen De humani 
corporis fabrica libri septem die ganze Grundlage der Medizin wieder in Frage. War der Mann überhaupt ernst zu nehmen? Galen in Zweifel ziehen! Hatte sein ehemaliger Pariser Lehrer Sylvius (Jacques Dubois, 1478-1555), der selber anatomisch tätig war und somit etwas von der Sache verstehen mußte ${ }^{3}$, nicht recht, diesen Vesalius «Vaesanus» (Vesanus) zu nennen ${ }^{4}$, den Wahnsinnigen, Überspannten und Betörten? Drohte denn die medizinische Welt ganz aus den Fugen zu geraten? Richtig, zwei Jahre vor dem Erscheinen der Fabrica war ja ein anderer Hitzkopf gestorben, Theophrastus Bombastus von Hohenheim, mit dem Übernamen Paracelsus. Auch er hatte versucht, die an den Hochschulen seit jeher gelehrte Medizin umzugestalten. Wie sollte bei all diesem In-Frage-Stellen althergebrachter Grundsätze und Lehren, über die nachgerade selbst die Fakultätskoryphäen miteinander zu zanken begannen, der praktizierende Arzt aus seiner Wissenschaft und Kunst noch klug werden? Wo blieb der ruhende Pol in der Erscheinungen Flucht, wie das «richtungsweisende Ziel» zu ersehen? Kaum ein anderer Arzt besaß das Format eines Felix Platter (1536-1614), dem es gelang, «einerseits als Arzt im Geist hippokratischer Medizin geschult, andererseits als Forscher vom Lehrgebäude Galens und vom Genius Vesals zutiefst erfüllt, ... in fast sechzigjähriger Praxis als anatomischer Lehrer, als Schöpfer der Anfänge von klinischer Anatomie, als Epidemiologe und Verfasser einer klassischen Kasuistik ein Lebenswerk, das seinesgleichen sucht», ${ }^{5}$ zu schaffen. Aber gerade diese Würdigung Platters deckt die geistige Zerfahrenheit auf, in der der damalige Arzt seinen Beruf ausübte. Wenn auch den einzelnen Arzt keine Schuld an der widerspruchsvollen Situation der Renaissancemedizin treffen mag, so waren es auch damals in erster Linie die Patienten, die unter den gegebenen Umständen zu leiden hatten. Einer dieser Patienten war Michel de Montaigne.

Montaigne (1533-1592) - «le Français le plus sage qui ait jamais existé» ${ }^{6}$ - gehört durch seine Essais ${ }^{7}$ und sein Journal de voyage en Italie par la Suisse et l'Allemagne en 1580 et $1581^{8}$ zu den immer aktuellen Autoren der Weltliteratur. Bezüglich seiner Aufnahme im deutschen Sprachbereich stellt der Basler Historiker Professor Herbert Lüthy freilich mit Recht fest, «daß Montaigne im deutschen Sprachbereich seit 1800 schlecht behandelt worden ist - und doch hätte ein Hauch Montaignescher Skepsis und Wahrhaftigkeit dem deutschen Geist sicherlich nur gut tun können». ${ }^{9}$ Diese Erwägungen treffen auch auf das Thema Montaigne und die Medizin zu. Wohl gibt es diesbezüglich eine Reihe von Studien besonders von französischen und angelsächsischen Ärzten; dennoch ist Montaignes Bedeutung in 
der Geschichte der Medizin noch nahezu unbekannt ${ }^{10}$. An diesem Umstand ist Montaigne vielleicht nicht ganz unschuldig. Denn er selbst ist mit der Medizin und den Ärzten nicht gerade wohlwollend umgegangen. «La vaine chose que c'est que la médecine» ${ }^{11}$, ruft er im Mai 1581 im italienischen Badeort della Villa bei Lucca enttäuscht aus. Eine gerechte Würdigung dieses strengen Urteils, das nicht sein einziges dieser Art ist, erfordert die Berücksichtigung von Montaignes Gesundheitszustand. Er litt nämlich seit 1577 an einem schweren Steinleiden der Harnwege; bei dieser damals lebensbedrohlichen Krankheit hatte er alle Gelegenheit, die Ohnmacht der Medizin seiner Zeit und das oft eigenartige Verhalten gewisser Ärzte am eigenen Leibe zu erfahren, wobei er zwischen den überheblichen Medikastern und den ehrlich-aufrichtigen Ärzten zu unterscheiden lernte.

Beiläufig sei darauf hingewiesen, daß Montaigne von seiner Lithiasis eine höchst anschauliche Nosographie gibt, die um so bemerkenswerter ist, als sie den natürlichen Verlauf einer Krankheit schildert, wie sie der heutige Arzt dank seinen therapeutischen Möglichkeiten kaum mehr zu Gesichte bekommt. Überhaupt zeugen viele Stellen in Montaignes Werken vom Interesse, das er der Medizin und ihren Problemen entgegenbrachte. Er hatte ja auch in jungen Jahren einigen medizinischen Unterricht bei Sylvius in Paris genossen, und später enthielt seine Bibliothek - «qui est des belles entre les librairies de village» ${ }^{12}$ - eine Anzahl medizinischer Werke. Auch erwies er anläßlich seines Basler Aufenthaltes im Herbst 1580 nicht dem Humanisten Thomas Platter (1499-1582) die Reverenz, sondern besuchte den Arzt Felix Platter (1536-1614) und sah sich dessen medizinische und naturkundliche Sammlungen aufmerksam an ${ }^{13}$.

Bei allem Interesse, das Montaigne der Medizin entgegenbrachte, stand er ihr dennoch skeptisch gegenüber. Eine Zusammenstellung der Argumente, die er gegen sie und die Ärzte ins Feld führte, ergäbe eine für die Kenntnis der Medizin des sechzehnten Jahrhunderts um so aufschlußreichere Arbeit, als Montaigne ein aufmerksamer und kritischer Beobachter seiner Zeitgenossen und der Zeitumstände war.

Er ließ es jedoch nicht bei seiner von polemischen Akzenten nicht immer ganz freien Kritik an der Medizin bewenden ${ }^{14}$. Den Mißständen stellt er ein Medizinprogramm entgegen, das einmal mehr zeigt, daß große Philosophen $^{15}$ «in der Zeit über der Zeit [stehen]. Jeder, auch der Größte, hat zwar seinen historischen Ort und trägt seine historischen Kleider. Das Kennzeichen der Größe aber ist, daß er nicht an sie gebunden scheint, sondern übergeschichtlich wird.» ${ }^{16}$ 
Der betreffende Text findet sich im zweiten Buch, Kapitel XXXVII, der Essais $^{17}$. Die Stelle lautet:

\begin{abstract}
«Or, si le mécompte du médecin est dangereux, il nous va bien mal, car il est bien malaisé qu'il n'y retombe souvent; il a besoin de trop de pièces, considérations et circonstances pour affûter ${ }^{\mathrm{a}}$ justement son dessein; il faut qu'il connaisse la complexion ${ }^{\mathrm{b}}$ du malade, sa température $^{\mathrm{c}}$, ses humeurs ${ }^{\mathrm{d}}$, ses inclinations, ses actions, ses pensements ${ }^{\mathrm{e}}$ mêmes et ses imaginations ${ }^{f}$; il faut qu'il se réponde des ${ }^{g}$ circonstances externes, de la nature du lieu, condition de l'air et du temps, assiette ${ }^{\text {h }}$ des planètes et leurs influences; qu'il sache en la maladie les causes, les signes, les affections ${ }^{i}$, les jours critiques; en la drogue, le poids, la force, le pays, la figure, l'âge, la dispensation ${ }^{j}$; et faut ${ }^{k}$ que toutes ces pièces ${ }^{1}$, il les sache proportionner et rapporter ${ }^{\mathrm{m}} \mathrm{l}^{\prime} u n e$ à l'autre pour en engendrer une parfaite symétrie. A quoi s'il faut ${ }^{\mathrm{n}}$ tant soit peu, si de tant de ressorts il y en a un tout seul qui tire à gauche ${ }^{\mathrm{o}}$, en voilà assez pour nous perdre. Dieu sait de quelle difficulté est la connaissance de la plupart de ces parties: car, pour exemple, comment trouvera-t-il le signe propre de la maladie, chacune étant capable d'un infini nombre de signes? Combien ont-ilsp de débats entre eux et de doutes sur l'interprétation des urines! Autrement d'où viendrait cette altercationq continuelle que nous voyons entre eux sur la connaissance du mal? Comment excuserionsnous cette faute, où ils tombent si souvent, de prendre martre pour renard? Aux maux que j'ai eus, pour peu qu'il y eût de difficulté, je n'en ai jamais trouvé trois d'accord.»
\end{abstract}

a) Disposer, ajuster. - b) Constitution. - c) Auch: tempérament. - d) Doppelsinn: Launen und Säfte. - e) Pensées et modes de penser. - f) Idées. - g) Qu'il connaisse les. - h) Position. i) Symptômes. - j) La manière de donner. - k) Il faut. - l) Parties, propriétés. - m) Mettre en rapport, adapter. - n) Se trompe. - o) S'écarte du droit chemin. - p) Les médecins. q) Discussion, échange de propos violents.

Dieser Text ist nicht nur in medizinischer Sicht bemerkenswert. Montaignes Absicht war ja weniger, ein lehrhaftes normatives Seinsollen auszuarbeiten, als vielmehr das reale Sosein des konkreten Menschen, seiner Lebensumstände und -gewohnheiten auf Grund eines empirisch gewonnenen Tatsachenmaterials zu erschließen ${ }^{18}$ :

«Les autres forment ${ }^{a}$ l'homme; je le récite $^{\mathrm{b}} .{ }^{19}$

a) Instruisent. - b) Décris.

«Je n'enseigne point; je raconte.» ${ }^{20}$

An dieser Arbeit wurde Montaigne zum großen Anthropologen. Im obigen Passus weicht er jedoch für einmal von seinem ursprünglichen Plan ab. Nicht mehr die kritische Darstellung des faktischen Menschen - hier des zeitgenössischen Arztes - steht nunmehr im Vordergrund ${ }^{21}$. Jetzt entwirft er mit Nachdruck ein in die Zukunft weisendes medizinisches Programm, das unhaltbar gewordene Traditionen ablösen soll. In seiner Skizze zeigt er die Prinzipien auf, ohne die es eine wissenschaftlich fundierte Medizin nicht 
geben kann. Eine Heilkunde, die nicht auf der detaillierten Anamnese, der exakten Untersuchung, der rational fundierten Kenntnis der Ätiologie und der Genese, der klaren Diagnose und dem genauen Wissen um die Eigenschaften und Wirkungen der eingeleiteten Therapie gründet, versinkt unweigerlich in ein abstruses Kurpfuschertum. Die Gefahr dieses Verfalls ist um so größer, als beim Fehlen wissenschaftlich begründeter Prinzipien die Ärzte eher einem fatalen Subjektivismus erliegen, wovon die Ärztekarikaturen und -satiren ein beredtes Zeugnis ablegen.

Es würde den Rahmen der vorliegenden Mitteilung überschreiten, allfälligen Vorläufern von Montaignes Postulaten innerhalb der Medizingeschichte nachzugehen. Wohl lassen sich in den Essais Reminiszenzen an hippokratische und galenische Schulen nachweisen. Der Tätigkeit ihrer Epigonen des sechzehnten Jahrhunderts steht Montaigne jedoch skeptisch gegenüber:

«Au demeurant, j'honore les médecins $[\ldots]$ pour l'amour d'eux-mêmes, en ayant vu
beaucoup d'honnêtes hommes et dignes d'être aimés. Ce n'est pas à eux que j'en veux, c'est
à leur art, et ne leur donne pas grand blâme de faire leur profit de notre sottise, car la
plupart du monde fait ainsi. Plusieurs vacations ${ }^{\mathrm{a}}$ et moindres et plus dignes que la leur
n'ont fondement et appui qu'aux abus publics.»" ${ }^{22}$
a) Professions.

Montaigne findet also auch auf Seite der Patienten eine Einstellung, die kurpfuscherischen Praktiken Vorschub leistet - ein gewiß noch heute aktuelles Thema.

Den medizinischen Übelständen seiner Zeit stellt Montaigne sein Programm gegenüber, das durch die Modernität seiner Forderungen besticht. Nicht mehr das einzelne Organ oder Organsystem ist finales Objekt der ärztlichen Tätigkeit, sondern die Persönlichkeit des kranken Menschen, d.h. das Ganze der Eigenschaften, die das Wesen der Person ausmachen. Durch den Einbezug der «pensements» und der «imaginations» des Patienten in die Anamnese ist Montaigne seiner Zeit weit voraus; er gehört zu den Vorläufern jener neueren Medizin, die sich in zunehmendem Maß um die zwischen Psyche und Soma bestehenden Wechselbezüge kümmert.

In diesem Zusammenhang sei bemerkt, daß die Essais nachgewiesenermaßen auf das Denken späterer Jahrhunderte eine nachhaltige Wirkung ausübten. Über dieses Thema gibt es eine reichhaltige Literatur. Besonders die Philosophen des siebzehnten und achtzehnten Jahrhunderts empfingen von Montaigne entscheidende Impulse ${ }^{23}$, die sich bei den zwischen Philoso- 
phie und Medizin bestehenden Beziehungen auf die Evolution der Medizin auswirkten $^{24}$.

Des weiteren ist zu beachten, daß Montaigne eine anregende Wirkung auf das Denken seiner Leser ausübt. Da es immer Ärzte gab, die sich in die Essais vertieften, ist die Frage nach Montaignes Einfluß auf die Medizin positiv zu beantworten. $\mathrm{Zu}$ den diesbezüglichen Texten dürfte auch der Abschnitt gehören, der den Gegenstand der vorliegenden Untersuchung bildet, auch wenn er in medizinischen Werken kaum ausdrücklich angeführt wurde.

\section{Anmerkungen}

${ }^{1}$ H.Buess, «Geleitwort» zu Jürg Wunderli, Das Geheimnis der Krankheit, Zürich [1967], S.11.

${ }^{2} \mathrm{Zu}$ diesem bedeutenden Renaissance-Arzt siehe Ernest Wickersheimer, «Les Médecins de la nation anglaise (ou allemande) de l'Université de Paris aux XIV et $\mathrm{XV}^{\mathrm{e}}$ siècles», Bulletin de la Société française d'Histoire de la Médecine, t. XII, Paris 1913, S.336-342; Marie-Louise Portmann, «Der Basler Humanistenarzt Wilhelm Copp (um 1460-1532)», Gesnerus 15 (1958) 106-119. - Bezüglich Cops Namen finden sich auch die Schreibweisen Copp, Cop(p)us, Le Cop.

${ }^{3}$ Sylvius ist in der Medizingeschichte vielleicht etwas vernachlässigt worden. Trotz einiger unangenehmer Charaktereigenschaften war er jedenfalls ein renommierter Arzt, der zahlreiche Schüler hatte und dem verschiedene Verdienste zukommen. So führte er in der Anatomie die Injektion der Gefäße ein, um sie besser sichtbar zu machen. Man zählt ihn zu den Pionieren der Psychiatrie; siehe René Semelaigne, Les Pionniers de la psychiatrie française avant et après Pinel, t. I, Paris 1930, S.17-21.

${ }^{4}$ So beispielsweise in der Schrift Vaesani cvivsdam calvmniarvm in Hippocratis Galenique rem anatomicam depulsio, per Iacobum Syluium ... Parrhisiis, Apud Catharinam Barbé, viduam Iacobi Gazelli, 1551.

${ }^{5}$ H.Buess, «Streiflichter auf die Geschichte der Medizinischen Fakultät Basel», Aktuelle Probleme aus der Geschichte der Medizin, Verhandlungen des XIX. Internationalen Kongresses für Geschichte der Medizin, herausgegeben von R. Blaser und H. Buess, Basel/New York 1966, S.397.

${ }^{6}$ C.-A.Sainte-Beuve, Nouveaux Lundis, t. II, Paris, Calmann-Lévy, s.d., «Montaigne en voyage», S.177. - Die Montaigne-Würdigung des bedeutenden französischen Literaturhistorikers Sainte-Beuve (1804-1869) wird immer noch gerne zitiert.

${ }^{7}$ Im vergangenen Jahr 1980 wurde das vierte Zentenarium des ersten Erscheinens der Essais weltweit gefeiert. An den internationalen Veranstaltungen trat die Ausstrahlungskraft dieses Buches einmal mehr zutage.

${ }^{8}$ Montaigne hatte sein Reisetagebuch nicht zur Veröffentlichung bestimmt. Das Manuskript wurde um 1770 in einer alten Truhe im Schloß Montaigne aufgefunden; die erste Publikation erfolgte 1774 . 
${ }^{9}$ Herbert Lüthy im Nachwort zu seiner partiellen Essais-Übertragung ins Deutsche, erschienen im Manesse-Verlag 1953, S.888.

${ }^{10}$ Es sei beispielsweise auf folgende Artikel hingewiesen: François Batisse, «Montaigne et les principes de la médecine expérimentale», in: Georges Palassie, Mémorial du $I^{\text {er }}$ Congrès international des Etudes Montaignistes (Bordeaux-Sarlat, $1^{\text {er }}-4$ Juin 1963), Bordeaux 1964, S.204-214; oder auf mein an der Montaigne-Jahrhundertfeier in Bordeaux im Juni 1980 gehaltenes Übersichtsreferat, «Aperçu sur la place de Montaigne dans l'histoire de la médecine», im Druck.

${ }^{11}$ Montaigne, Journal de voyage en Italie, édition présentée, établie et annotée par Pierre Michel, Le Livre de poche [1974], S. 383.

${ }^{12}$ Essais, 2.Buch, Kapitel XVII. - Die Essais-Zitate sind der in moderne französische Orthographie übertragenen, von Pierre Michel bei Gallimard 1965 in der «Collection Folio» edierten Ausgabe entnommen.

${ }^{13}$ In einem an den «Journées Montaigne, Mulhouse/Bâle» (Oktober 1980) gehaltenen Referat bin ich näher auf Montaignes Aufenthalt in Basel eingegangen. Die Arbeit befindet sich im Druck.

${ }^{14}$ Zum Beispiel: «Je consulte peu des altérations ${ }^{\mathrm{a}}$ que je sens, car ces gens-ci ${ }^{\mathrm{b}}$ sont avantageux $^{\mathrm{c}}$ quand ils vous tiennent à leur miséricorde ${ }^{\mathrm{d}}$; ils vous gourmandent les oreilles de leurs prognostics; et, me surprenant autrefois affaibli du mal, m'ont injurieusement traitée de leurs dogmes et trogne magistrale, me menaçant tantôt de grandes douleurs, tantôt de mort prochaine.» Essais, 3. Buch, Kapitel XIII.

a) Je consulte peu les médecins au sujet des altérations de santé. - b) Les médecins. - c) Font les supérieurs. - d) A leur merci. - e) M'ont maltraité.

${ }^{15}$ In seinem Werk Die großen Philosophen, München [1959], S.48, reiht Karl Jaspers Montaigne unter die großen Philosophen ein.

${ }^{16}$ Id., a. a. O., S. 39.

${ }^{17}$ Im zweiten Band der Essais-Edition von Pierre Michel, S. 554.

${ }^{18}$ Siehe hierzu Hugo Friedrich, Montaigne, zweite neubearbeitete Auflage, Bern und München [1967], 1. Kapitel und an anderen Stellen.

${ }^{19}$ Essais, 3. Buch, Kapitel II.

${ }^{20}$ Essais, a.a.O.

${ }^{21}$ Montaigne läßt seiner Kritik an den Ärzten und der Medizin an vielen Stellen der Essais freien Lauf, besonders im Kapitel XXXVII des zweiten Buches. - Zu diesem Thema siehe auch G.C.Donadi, «Montaigne, o della polemica medica», Aktuelle Probleme aus der Geschichte der Medizin, a. a. O., S.537-540.

${ }^{22}$ Essais, 2. Buch, Kapitel XXXVII.

${ }^{23}$ Eigenartigerweise wurden diese Zusammenhänge im deutschen Schrifttum wenig beachtet.

${ }^{24}$ Einige diesbezügliche Hinweise finden sich in meiner Untersuchung «Descartes' Grundgedanken in medizinhistorischer Sicht», Gesnerus 35 (1978) 44-53.

\section{Résumé}

Dans un passage condensé du chapitre II, xxxvii, «De la ressemblance des enfants aux pères», des Essais, sont exposés les principes d'une médecine globale de la personne qui témoignent de l'actualité toujours présente de Montaigne aussi dans le domaine de la médecine. 


\section{Summary}

The 16th century was a time of revolution in medicine (Paracelsus and Vesalius). The great French philosopher Michel de Montaigne has - in a passage of his "Essais"-exposed the principles of a good medicine. These remarks show, once more, the actuality of Montaigne's thoughts.

Ehrendozent Dr.med. et phil. René Bernoulli

Amselstraße 35

4059 Basel 